University of New Orleans

ScholarWorks@UNO

1995

\title{
Prediction of signal-to-noise ratio gain for passive higher-order correlation detection of energy transients
}

\author{
Lisa A. Pflug \\ George E. loup \\ University of New Orleans \\ Juliette W. Ioup \\ University of New Orleans \\ Robert L. Field
}

Follow this and additional works at: https://scholarworks.uno.edu/phys_facpubs

Part of the Physics Commons

\section{Recommended Citation}

J. Acoust. Soc. Am. 98, 248 (1995)

This Article is brought to you for free and open access by the Department of Physics at ScholarWorks@UNO. It has been accepted for inclusion in Physics Faculty Publications by an authorized administrator of ScholarWorks@UNO.

For more information, please contact scholarworks@uno.edu. 


\title{
Prediction of signal-to-noise ratio gain for passive higher-order correlation detection of energy transients
}

\author{
Lisa A. Pflug \\ Naval Research Laboratory, Stennis Space Center, Mississippi 39529-5004
}

George E. loup and Juliette W. loup

Department of Physics, University of New Orleans, New Orleans, Louisiana 70148

Robert L. Field

Naval Research Laboratory, Stennis Space Center, Mississippi 39529-5004

(Received 3 December 1993; revised 30 August 1994; accepted 2 February 1995)

\begin{abstract}
In general, higher-order correlation detectors perform well in passive detection for signals of high third- and fourth-order moments. Previous studies by the authors have shown that the normalized third- and fourth-order signal moments are reliable indicators of higher-order correlation detector performance [Pflug et al. (1992b)]. For a deterministic energy transient of known moments through fourth order, it is possible to predict theoretically the amount of gain over an ordinary cross-correlation detector for a bicorrelation or tricorrelation detector applied in a noise environment of known variance. In this paper, formulas that predict detector performance for passive detection at the minimum detectable level are derived. The noise is assumed to be stationary and zero mean with Gaussian correlation central ordinate probability density functions. To test the formulas, SNR detection and gain curves are generated using hypothesis testing and Monte Carlo simulations on a set of test signals. The test signals are created by varying the time width of a pulse-like signal in a sampling window of fixed time duration, resulting in a set of test signals with varying signal moments. Good agreement is found between the simulated and theoretical results. The effects of observation time (length of detection window) and sampling interval on detector performance are also discussed and illustrated with computer simulations. The prediction formulas indicate that decreasing the observation time or the sampling interval (assuming the signal is sufficiently sampled and the detection window contains the entire signal) improves detection performance. However, the rate of improvement is different for the three detectors. The SNR required to achieve the minimum detectable level of detection performance at a given probability of false alarm $\left(P_{\mathrm{fa}}\right)$ decreases with the fourth root of the observation time and sampling interval for the cross-correlation detector, the sixth root for the bicorrelation detector, and the eighth root for the tricorrelation detector. Relative detector performance also varies with $P_{\mathrm{fa}}$. The probability of detection $\left(P_{d}\right)$ for higher-order detectors degrades less rapidly with decreasing $P_{\mathrm{fa}}$ than the $P_{d}$ for ordinary correlations. Thus higher-order correlators can be especially appropriate when a very low $P_{\mathrm{fa}}$ is required.
\end{abstract}

PACS numbers: $43.60 . \mathrm{Gk}, 43.60 . \mathrm{Cg}$

\section{INTRODUCTION}

For transients of high skewness and kurtosis, it has been shown that higher-order spectral detectors can outperform second-order or energy detectors in passive detection, i.e., the unknown source model. The higher-order frequencydomain detection methods proposed by Kletter and Messer (1989) and Hinich and Wilson (1990) are applicable to stationary random signals. These methods use segmentation for averaging which results in noise suppression. However, segmentation of short-time energy transient signals is generally not appropriate. The problem addressed in the current paper is that of detecting a transient for which only one short-time realization (received on multiple sensors) is available for processing. Not only are the signals short, in that not many samples are available for segmentation, but changes in the signal over its duration also make segmentation inappropriate. [The issue of averaging over the sensors is addressed by
Pflug et al. (1994).] Hinich (1990) addresses this problem in a paper proposing a frequency-domain method of transient detection in Gaussian noise using the inner triangle of the smoothed bispectrum. Care must be taken in applying the altemate detection test described by Hinich based on the outer triangle, which holds for calculations done with continuous-time transients and not for calculations done with discrete-time transients (Pflug et al., 1993, Appendix B). The authors have shown that higher-order time-domain detection methods for transient signals can show improvement over the ordinary second-order, or cross-correlation, method (Ioup et al., 1989a, b, 1991, 1993; Pflug et al., 1989, 1990a, b, 1992b, 1994).

In earlier work the authors have described studies using Monte Carlo simulations and hypothesis testing, and the resulting receiver operating characteristic (ROC) curves to examine higher-order correlation detection performance of transient signals distorted by Gaussian noise. In this paper, 
prediction formulas that allow theoretical evaluation of higher-order correlation passive detection performance are derived.

The efficacy of higher-order detectors vis-à-vis the ordinary correlation detector has been a matter of continuing interest. It is still under discussion whether it is possible for a higher-order detector to do better than a matched filter in the active case. The appropriate approach to answer these questions is the derivation of theoretical formulas which describe performance for the passive and active cases. Then the circumstances under which the higher-order detectors do better than the ordinary correlation and those signal properties and detection conditions which give superiority for the higher-order detectors can be clearly delineated.

The prediction formulas require knowledge of the noise variance and the low-order moments of a sampled energy signal and give the signal-to-noise ratio (SNR) at which the minimum detectable level is achieved for a given probability of false alarm $\left(P_{\mathrm{F}_{3}}\right)$. This enables ranking of the detectors under various test conditions. From these formulas, one can predict the amount of theoretical SNR gain that a higherorder correlation detector can provide over the crosscorrelation detector. In addition, for a zero-mean signal, one can use the theoretical expressions to calculate the minimum levels of signal skewness and kurtosis for which the bicorrelation and tricorrelation detectors will show improvement over the cross correlation detector. Prediction formulas to determine detector performance for transient signals also depend on factors such as the observation time, sampling rate, and $P_{\mathrm{fa}}$, which are discussed in detail.

One important application of passive detection is in underwater acoustics. An environmental model can be used to identify regions of multipath distortion where the signal third- and fourth-order moments remain high, and for which selected levels of SNR gain may be expected. A preliminary study by Field and Leclere (1993) shows examples of the robustness of a finite-element parabolic equation propagation model in predicting signal kurtosis of multipath signals, and thus tricorrelation detection performance. Using real and simulated data, they find that although signal kurtosis in general decreases with increasing multipath distortion, there exist ocean areas, particularly near the surface and bottom, where signal kurtosis remains high and tricorrelation detection may be applicable.

Higher-order correlation detectors are described in Sec. I. In Sec. II, non-normalized and normalized moments for energy transients and the second moment for stationary random signals, such as noise, are given. Theoretical detector performance formulas and SNR gain formulas are derived in Sec. III. In Sec. IV, discussions concerning detector dependence on $P_{\mathrm{fa}}$, sampling interval, and observation time are presented. Finally, in Sec. V, computer simulations are used to test the theoretical prediction formulas. Conclusions are given in Sec. VI.

\section{CORRELATION DETECTORS}

The ordinary correlation detector for an unknown source signal involves the cross-correlation of received data from two sensors, which is defined for discrete-time energy signals as (Bracewell, 1986)

$$
c_{2}(\tau)=\Delta t \sum_{k=0}^{N-1} r_{1}(t) r_{2}(t+\tau),
$$

where $t=k \Delta t$ and $\tau=j \Delta t, r_{i}(t)$ represents a received signal of the form $r_{i}(t)=s(t)+n_{i}(t), s(t)$ represents an energy signal, and each $n_{i}(t)$ represe tts one noise realization. When the noise-free signal reaching each sensor is different, $s(t)$ must be replaced by $s_{i}(t)$. The cross-correlation detector compares the cross-correlation central ordinate value of the two noisy received signals to a preset threshold. Received signals from three sensors may be correlated similarly to form the bicorrelation (Ioup et al., 1989b)

$$
c_{3}\left(\tau_{1}, \tau_{2}\right)=\Delta t \sum_{k=0}^{N-1} r_{1}(t) r_{2}\left(t+\tau_{1}\right) r_{3}\left(t+\tau_{2}\right),
$$

or from four sensors to form the tricorrelation (Ioup et al., 1989b)

$$
\begin{aligned}
c_{4}\left(\tau_{1}, \tau_{2}, \tau_{3}\right)= & \Delta t \sum_{k=0}^{N-1} r_{1}(t) r_{2}\left(t+\tau_{1}\right) r_{3}\left(t+\tau_{2}\right) \\
& \times r_{4}\left(t+\tau_{3}\right),
\end{aligned}
$$

with the corresponding central (or other) ordinate threshold detectors defined. The bicorrelation and tricorrelation can also be formed using output from only two sensors and repeating signals in various ways (Pflug et al., 1992b, 1994).

\section{MOMENTS FOR SAMPI.ED ENERGY AND STATIONARY RANDOM SIGNALS}

A signal is said to be an energy signal if it has finite sum of squares. That is, for a signal $x(t)$ and $t=k \Delta t$,

$$
\sum_{k=-\infty}^{\infty} x^{2}(t) \Delta t<\infty .
$$

In contrast, a power signal (such as the noise modeled in this paper) has finite power, or

$$
0<\lim _{N \rightarrow \infty} \frac{1}{(2 N+1) \Delta t} \sum_{k=-N}^{N^{\prime}} x^{2}(t) \Delta t<\infty .
$$

That is, a power signal sum is finite only if the sum is normalized by time (Robinson, 1980).

The second-, third-, and fourth-order non-normalized moments for a discrete-time energy signal $s=\left\{s_{0}, s_{1}, \ldots, s_{N-1}\right\}$ are the same as the correlations given in the previous section. However, only the central ordinate values of the energy signal moments are needed for detection at zero lag, and these are given by

$$
m_{p}^{s}=\Delta t \sum_{k=0}^{N-1} s^{p}(t)
$$


where $p=1,2,3, \ldots$ represents the order of correlation, and $m_{p}^{s}$ the central ordinate value of the correlation. For sufficient sampling and assuming the observation window contains the entire signal, these values are independent of both sampling interval $(\Delta t)$ and observation time $(T)$, and are thus good descriptors for a deterministic bandlimited energy transient. However, non-normalized signal moments do not always give the simplest formulas for central ordinate correlation detector performance since the noise is a power signal. To improve compatibility when combining representations for energy and power signals, the energy signal moments may also be defined using normalizations analogous to traditional power signal normalizations. However, the signal duration $T_{s}$ is used in the normalization instead of $T$ to avoid the signal moments and SNR changing with observation time. $T_{s}$ is a signal property just as the moments are. A natural choice for $T_{s}$ is the shortest duration which includes all nonzero values of the signal.

Normalized signal moments can be defined simply by subtracting the means and dividing the moment definition of Eq. (6) by $T_{s}$. If it is desired to have dimensionless thirdand fourth-order moments, which are zero for Gaussian distributed ordinate values, then the mean, variance, skewness, and kurtosis for finite-energy signals, denoted by $\bar{s}, \sigma_{s}^{2}, S$, and $K$, respectively, are defined by Press et al. (1986) as

$$
\begin{aligned}
& \bar{s}=\frac{\Delta t}{T_{s}} \sum_{k=0}^{N-1} s(t), \\
& \sigma_{s}^{2}=\frac{\Delta t}{T_{s}} \sum_{k=0}^{N-1}[s(t)-\bar{s}]^{2}, \\
& S=\frac{\Delta t}{T_{s}} \sum_{k=0}^{N-1}\left[\frac{s(t)-\bar{s}}{\sigma_{s}}\right]^{3}, \\
& K=\left\{\frac{\Delta t}{T_{s}} \sum_{k=0}^{N-1}\left[\frac{s(t)-\bar{s}}{\sigma_{s}}\right]^{4}\right\}-3 .
\end{aligned}
$$

For zero-mean signals, the first four normalized signal moments defined in Eqs. (7)-(10) can be written as

$$
\begin{aligned}
& \bar{s}=m_{1}^{s} / T_{s}, \\
& \sigma_{s}^{2}=m_{2}^{s} / T_{s}, \\
& S=m_{3}^{s} \sqrt{T_{s}} /\left(m_{2}^{s}\right)^{3 / 2}, \\
& K=\left\{m_{4}^{s} T_{s} /\left(m_{2}^{s}\right)^{2}\right\}-3 .
\end{aligned}
$$

Like the non-normalized signal moments, the normalized signal moments are independent of sampling interval and observation time.

Predicting detector performance requires knowledge of the noise variance in addition to the signal moments. We assume stationary zero-mean noise, which is a power signal, with an ensemble average to define the sample variance, or, if ergodicity and a finite sum approximation are assumed,

$$
\sigma_{n}^{2}=\frac{\Delta t}{T} \sum_{k=0}^{N-1} n(t)^{2}=\frac{1}{N} \sum_{k=0}^{N-1} n(t)^{2} .
$$

Note that the time definition contains a normalization by the observation time while the energy signal variance contains a normalization by the signal duration. In this way, both the signal and noise statistics remain unaffected by changes in sampling rate and observation time. All the calculations in this paper are based on finite-time realizations. The differences between the sample and population noise means and variances are assumed to be small for comparison of theoretical and simulated results. $\sigma_{n}^{2}$ is used to represent both variances in this paper.

We take SNR of an energy signal realization in noise to be

$$
\mathrm{SNR}=\sigma_{s} / \sigma_{n} .
$$

However, rewriting the signal variance in Eq. (8) in the form

$$
\begin{aligned}
\sigma_{s}^{2}=\frac{\Delta t}{T_{s}} \sum_{k=0}^{N-1}[s(t)-\bar{s}]^{2} & =\frac{\Delta t}{T_{s}} \sum_{k=0}^{N-1}\left[s(t)^{2}-\bar{s}^{2}\right] \\
& =\frac{\Delta t}{T_{s}} \sum_{k=0}^{N-1}\left[s(t)^{2}-\left(\frac{m_{1}^{s}}{T_{s}}\right)^{2}\right] \\
& =\frac{1}{T_{s}}\left[m_{2}^{s}(0)-\frac{m_{1}^{s^{2}}}{T_{s}}\right]
\end{aligned}
$$

allows us to define SNR as

$$
\mathrm{SNR}=\frac{1}{\sigma_{n}} \sqrt{\frac{1}{T_{s}}\left[m_{2}^{s}-\frac{m_{1}^{s^{2}}}{T_{s}}\right] .}
$$

For a zero-mean signal, the expression for SNR simplifies to

$$
\mathrm{SNR}=\sqrt{m_{2}^{s}(0)} / \sigma_{n} \sqrt{T_{s}} .
$$

In Eqs. (18) and (19), SNR is shown in forms that exhibit the dependence of SNR on the signal moments and duration. Note that even though the noise-free original signal may have a shorter time duration than the noisy received signal, the SNR is independent of $T$. In this paper, SNR is converted to power $\mathrm{dB}$ using $20 \log _{10}\left(\sigma_{s} / \sigma_{n}\right)=10 \log _{10}\left(\sigma_{s}^{2} / \sigma_{n}^{2}\right)$ for numerical examples and gains.

\section{THEORETICAL EVALUATION OF DETECTOR PERFORMANCE}

Given a fixed probability of detection, $P_{d}$, of 0.5 , also known as the minimum detectable level, and a selected probability of false alarm, $P_{\text {fa }}$, one can calculate the SNR required for ordinary and higher-order correlation detectors and the probable gain, or improvement, in SNR that a bicorrelation or tricorrelation detector will show over an ordinary cross-correlation detector for an energy signal. This is done using the statistics of the signal-absent and signal-present probability density functions (PDFs) which define a ROC curve. We assume the noise is zero mean, independent of the signal, and satisfies the assumptions in Appendix A. We also assume that for sufficiently large ensembles, the PDFs of the signal-absent correlation central ordinate values are Gaussian (see Appendix B) and that their areas can be calculated numerically or found in standard tables. See Van Trees (1968) 
and Egan (1975) for detailed descriptions of ROC curves, and Pflug et al. (1992b) for details on the simulations used later in this paper.

The assumption is made that the signal-present PDF is symmetric and centered at the signal ordinate of detection, e.g., for an ordinary correlation detector it is at the correlation central ordinate value of the noise-free signal, $m_{2}^{s}$. Thus the threshold of detection corresponding to $P_{d}=0.5$ is also $m_{p}^{s}$. If this is not the case, then the median of the PDF must be determined to get the threshold.

For the cross-correlation detector,

$$
z_{n}^{\mathrm{CC}}=\left(m_{2}^{s}-\mu_{2}^{n}\right) / \sqrt{\alpha_{n}^{2}},
$$

where $\mu_{2}^{n}$ represents the mean and $\alpha_{n}^{2}$ the variance of the PDF of the ensemble of signal-absent cross-correlation central ordinate values. The $z_{n}$ score of Eq. (20) defines the level of $P_{\mathrm{fa}}\left(\right.$ Egan, 1975). For zero-mean noise, $\mu_{2}^{n}=0$. A similar analysis is done for the bicorrelation detector at the minimum detectable level. Evaluating the $z_{n}$ score at the signalpresent PDF mean, $m_{3}^{s}$, results in

$$
z_{n}^{\mathrm{BC}}=\left(m_{3}^{s}-\mu_{3}^{n}\right) / \sqrt{\beta_{n}^{2}} .
$$

$\mu_{3}^{n}$ and $\beta_{n}^{2}$ represent the mean and variance, respectively, of the PDF of the ensemble of signal-absent bicorrelation central ordinate values, and $\mu_{3}^{n}$ is zero for zero-mean noise. The signal-present PDF of the ensemble of central ordinate values of the tricorrelation of four different signal-plus-noise realizations is centered at the central ordinate value of the noise-free signal autotricorrelation, $m_{4}^{s}$. Thus for the minimum detectable level the $z_{n}$ score is

$$
z_{n}^{\mathrm{TC}}=\left(m_{4}^{s}-\mu_{4}^{n}\right) / \sqrt{\gamma_{n}^{2}},
$$

where $\mu_{4}^{n}$ is the mean and $\gamma_{n}^{2}$ is the variance of the ensemble of signal-absent tricorrelation central ordinate values. For noise with a nonzero-mean Gaussian PDF of correlation central ordinate values, $\mu_{2}^{n}, \mu_{3}^{n}$, and $\mu_{4}^{n}$ would be nonzero. The zero-mean PDF assumption is made only for simplicity.

\section{A. Ensemble variances}

To derive the prediction formulas, the necessary as sumptions concerning the noise process are in Appendix A for the $p$ th-order correlation. Evaluation of the $z_{n}$ scores for the cross-correlation, bicorrelation, and tricorrelation requires evaluation of the signal-absent PDF variance for each of the three correlations.

The ensemble variance of an infinite number of crosscorrelation central ordinate values of two finite-length noise realizations, $n_{a}$ and $n_{b}$, is evaluated using the expectation operator

$$
\begin{aligned}
\alpha_{n}^{2}= & E\left\{\left[\sum_{k=0}^{N-1} n_{a}(t) n_{b}(t) \Delta t\right]^{2}\right\} \\
& -E^{2}\left\{\sum_{k=0}^{N-1} n_{a}(t) n_{b}(t) \Delta t\right\} .
\end{aligned}
$$

Since the noise is uncorrelated and zero mean, the second term is approximately zero and ignored. Then,

$$
\begin{aligned}
\alpha_{n}^{2} & =E\left\{\left[\Delta t \sum_{k_{1}=0}^{N-1} n_{a}\left(t_{1} n_{b}\left(t_{1}\right)\right]\left[\Delta t \sum_{k_{2}=0}^{N-1} n_{a}\left(t_{2}\right) n_{b}\left(t_{2}\right)\right]\right\}\right. \\
& =E\left\{(\Delta t)^{2} \sum_{k_{1}=0}^{N-1} \sum_{k_{2}=c}^{N-1} n_{a}\left(t_{1}\right) n_{a}\left(t_{2}\right) n_{b}\left(t_{1}\right) n_{b}\left(t_{2}\right)\right\} \\
& =(\Delta t)^{2} E\left\{\sum_{k_{1}=0}^{N-1} n_{a}^{2}\left(t_{1}\right) n_{b}^{2}\left(t_{1}\right)\right\} \\
& =(\Delta t)^{2} \sum_{k=0}^{N-1} E\left\{n_{a}^{2}(t) n_{b}^{2}(t)\right\} \\
& =(\Delta t)^{2} N E\left\{n_{a}^{2}(t)\right\} E\left\{n_{b}^{2}(t)\right\} \\
& =(\Delta t)^{2} N \sigma_{n}^{4} \\
& =T \Delta t \sigma_{n}^{4} .
\end{aligned}
$$

The ensemble variance of the bicorrelation central ordinate values of three finite-length noise realizations is

$$
\begin{aligned}
\beta_{n}^{2}= & E\left\{\left[\sum_{k=0}^{N-1} n_{a}(t) n_{b}(t) n_{c}(t) \Delta t\right]^{2}\right\} \\
& -E^{2}\left\{\sum_{k=0}^{N-1} n_{a}(t) n_{b}(t) n_{c}(t) \Delta t\right\} .
\end{aligned}
$$

Since the second term is approximately zero, it can be ignored:

$$
\begin{aligned}
& \beta_{n}^{2}= E\left\{\left[\sum_{k_{1}=0}^{N-1} n_{a}\left(t_{1}\right) n_{b}\left(t_{1}\right) n_{c}\left(t_{1}\right)\right]\right. \\
&\left.\times\left[\sum_{k_{2}=0}^{N-1} n_{a}\left(t_{2}\right) n_{b}\left(i_{2}\right) n_{c}\left(t_{2}\right)\right]\right\} \\
&= E\left\{(\Delta t)^{2} \sum_{k_{1}=0}^{N-1} \sum_{k_{2}=0}^{N-1} n_{a}\left(t_{1}\right) n_{a}\left(t_{2}\right) n_{b}\left(t_{1}\right)\right. \\
&\left.\times n_{b}\left(t_{2}\right) n_{c}\left(t_{1}\right) n_{c}\left(i_{2}\right)\right\} \\
&(\Delta t)^{2} E\left\{\sum_{k_{1}=0}^{N-1} n_{a}^{2}\left(t_{1}\right) n_{b}^{2}\left(t_{1}\right) n_{c}^{2}\left(t_{1}\right)\right\} \\
&=(\Delta t)^{2} \sum_{k=0}^{N-1} E\left\{n_{a}^{2}(t) n_{b}^{2}(t) n_{c}^{2}(t)\right\} \\
&=(\Delta t)^{2} N E\left\{n_{a}^{2}(t)\right\} E\left\{n_{b}^{2}(t)\right\} E\left\{n_{c}^{2}(t)\right\} \\
&= T \Delta t \sigma_{n}^{6} .
\end{aligned}
$$

The ensemble variance of the tricorrelation central ordinate values of four finite-length noise realizations is

$$
\begin{aligned}
\gamma_{n}^{2}= & E\left\{\left[\sum_{k=0}^{N-1} n_{a}(t) n_{b}(t) n_{c}(t) n_{d}(t) \Delta t\right]^{2}\right\} \\
& -E^{2}\left\{\sum_{k=0}^{N-1} n_{a}(t) n_{b}(t) n_{c}(t) n_{d}(t) \Delta t\right\} .
\end{aligned}
$$


For zero-mean noise which is uncorrelated in quadruples (Appendix A), the expression reduces to

$$
\begin{aligned}
\gamma_{n}^{2} & =(\Delta t)^{2} \sum_{k=0}^{N-1} E\left\{n_{a}(t)^{2} n_{b}(t)^{2} n_{c}(t)^{2} n_{d}(t)^{2}\right\} \\
& =T \Delta t \sigma_{n}^{8} .
\end{aligned}
$$

\section{B. Performance prediction formulas}

The ensemble variances can be used in the $z_{n}$ scores given previously to obtain detection prediction formulas. Starting with the $z_{n}$ score (corresponding to the selected $P_{\mathrm{f} a}$ ) for the cross-correlation detector given in Eq. (20),

$$
z_{n}^{\mathrm{CC}}=m_{2}^{s} / \sqrt{\alpha_{n}^{2}}=m_{2}^{s} / \sigma_{n}^{2} \sqrt{T \Delta t} .
$$

This can be recast into a formula containing SNR:

$$
z_{n}^{\mathrm{CC}}=\frac{\sigma_{s}^{2} \sqrt{T \Delta t}}{m_{2}^{s}}\left[\mathrm{SNR}_{\mathrm{CC}}\right]^{2}
$$

or

$$
\mathrm{SNR}_{\mathrm{CC}}=\sigma_{s} \sqrt{z_{n}^{\mathrm{CC}} \sqrt{T \Delta t} / m_{2}^{s}},
$$

in which $\mathrm{SNR}_{\mathrm{CC}}$ represents the SNR required to achieve the predefined level of detection of $P_{d}=0.5$ and selected $P_{\mathrm{fa}}$.

Bicorrelation detector performance is derived by substituting $\beta_{n}^{2}=T \Delta t \sigma_{n}^{6}$ into Eq. (21) to give

$$
z_{n}^{\mathrm{BC}}=m_{3}^{s} / \sqrt{\beta_{n}^{2}}=m_{3}^{s} / \sigma_{n}^{3} \sqrt{T \Delta t} .
$$

To show the SNR dependence, rewrite the equation as

$$
z_{n}^{\mathrm{BC}}=\frac{\sigma_{s}^{3} \sqrt{T \Delta t}}{m_{3}^{s}}\left[\mathrm{SNR}_{\mathrm{BC}}\right]^{3}
$$

or

$$
\mathrm{SNR}_{\mathrm{BC}}=\sigma_{s} \sqrt[3]{z_{n}^{\mathrm{BC}} \sqrt{T \Delta t} / m_{3}^{s}} .
$$

Similarly, by substituting Eq. (25c) into Eq. (22), the tricorrelation detector prediction formula is given by

$$
z_{n}^{\mathrm{TC}}=\frac{m_{4}^{s}}{\sqrt{\gamma_{n}^{2}}}=\frac{m_{4}^{s}}{\sigma_{s}^{4} \sqrt{T \Delta t}}\left[\mathrm{SNR}_{\mathrm{TC}}\right]^{4}
$$

or

$$
\mathrm{SNR}_{\mathrm{TC}}=\sigma_{s} \sqrt[4]{z_{n}^{\mathrm{TC}} \sqrt{T \Delta t} / m_{4}^{s}} .
$$

The prediction formulas simplify even more for zeromean energy signals. Using Eq. (19), the cross-correlation SNR reduces to

$$
\mathrm{SNR}_{\mathrm{CC}}=\sqrt{\frac{m_{2}^{s}}{T_{s}}} \sqrt{\frac{z_{n}^{\mathrm{CC}} \sqrt{T \Delta t}}{m_{2}^{s}}}=\sqrt{\frac{z_{n}^{\mathrm{CC}} \sqrt{T \Delta t}}{T_{s}}} .
$$

The bicorrelation and tricorrelation reduce to forms that can be easily written in terms of signal skewness and kurtosis, respectively. Thus

$$
\mathrm{SNR}_{\mathrm{BC}}=\sqrt[3]{\frac{z_{n}^{\mathrm{BC}} \sqrt{T \Delta t}\left(m_{2}^{s}\right)^{3 / 2}}{T_{s}^{3 / 2} m_{3}^{s}}}=\sqrt[3]{\frac{z_{n} \sqrt{T \Delta t}}{T_{s} S}}
$$

and

$$
\mathrm{SNR}_{\mathrm{TC}}=\sqrt[4]{\frac{z_{n}^{\mathrm{TC}} \sqrt{T \Delta t}\left(m_{2}^{s}\right)^{2}}{T_{s}^{2} m_{4}^{s}}}=\sqrt[4]{\frac{z_{n} \sqrt{T \Delta t}}{T_{s}(K+3)}} .
$$

Only the signal-present and signal-absent PDFs of correlation central ordinate values are required to derive general prediction formulas for energy transients in noise. In particular, at the minimum detectable level, only the signal-absent PDF and the median of the signal-present PDF are required. Appendix B gives the assumptions necessary for the signalabsent correlation central ordinate values of the noise to have a Gaussian density and shows that the moments of the PDFs are consistent with a Gaussian density given these assumptions.

\section{SNR gain formulas}

For a given $P_{\mathrm{fa}}\left(z_{n}^{\mathrm{CC}}=z_{n}^{\mathrm{BC}}=z_{n}\right)$, the SNR gain of the bicorrelation detector over the cross-correlation detector at the minimum detectable level in $\mathrm{dB}$ is defined by $20 \log \mathrm{SNR}_{\mathrm{CC}}-20 \log \mathrm{SNR}_{\mathrm{BC}}$. This is equivalent to

$20 \log \left[\frac{\sqrt{z_{n} \sqrt{T \Delta t} / m_{2}^{s}}}{\sqrt[3]{z_{n} \sqrt{T \Delta t} / m_{3}^{s}}}\right]=20 \log \left[\frac{z_{n}^{1 / 6}(T \Delta t)^{1 / 12}\left(m_{3}^{s}\right)^{1 / 3}}{\left(m_{2}^{s}\right)^{1 / 2}}\right]$.

For zero-mean energy signals, the bicorrelation SNR gain can be expressed in terms of skewness as

$$
20 \log \left[\frac{\sqrt{z_{n} \sqrt{T \Delta t} / T_{s}}}{\sqrt[3]{z_{n} \sqrt{T \Delta t} / T_{s} S}}\right]=20 \log \left[\frac{z_{n}^{2}(T \Delta t) S^{4}}{T_{s}^{2}}\right]^{1 / 12} .
$$

Similarly, the tricorrelation SNR gain in $\mathrm{dB}$, $20 \log \mathrm{SNR}_{\mathrm{CC}}-20 \log \mathrm{SNR}_{\mathrm{TC}}$, for nonzero-mean signals at a fixed $P_{\mathrm{fa}}\left(z_{n}^{\mathrm{CC}}=z_{n}^{\mathrm{TC}}=z_{n}\right)$, is given by

$20 \log \left[\frac{\sqrt{z_{n} \sqrt{T \Delta t} / m_{2}^{s}}}{\sqrt[4]{z_{n} \sqrt{T \Delta t} / m_{4}^{s}}}\right]=20 \log \left[\frac{z_{n}^{1 / 4}(T \Delta t)^{1 / 8}\left(m_{4}^{s}\right)^{1 / 4}}{\left(m_{2}^{s}\right)^{1 / 2}}\right]$.

For zero-mean energy signals, the tricorrelation SNR gain is expressed as

$$
\begin{aligned}
20 \log \left[\frac{\sqrt{z_{n} \sqrt{T \Delta t} / T_{s}}}{\sqrt[4]{z_{n} \sqrt{T \Delta t} / T_{s}(K+3)}}\right] \\
=20 \log \left[\frac{z_{n}^{2}(T \Delta t)(K+3)^{2}}{T_{s}^{2}}\right]^{1 / 8} .
\end{aligned}
$$

For a given observation time and sampling rate, the bicorrelation or tricorrelation SNR gain at $P_{d}=0.5$ can be determined for any $P_{\mathrm{fa}}$.

For example, using sampling interval $\Delta t=1 / 1024 \mathrm{~s}$ and $T=T_{s}=2 \mathrm{~s}$, the predicted bicorrelation and tricorrelation SNR gains as a function of signal skewness and kurtosis for a zero-mean energy signal are given by the curves in Fig. 1 for two values of $P_{\mathrm{fa}}$. It is evident from these curves that a decrease in $P_{\mathrm{fa}}$ (increase in $z_{n}$ ) corresponds to an increase in 

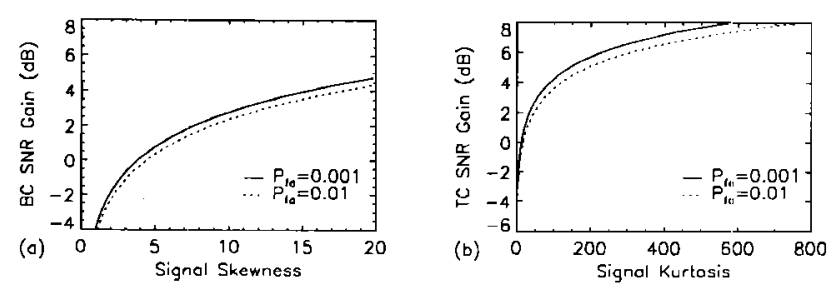

FIG. 1. Theoretical bicorrelation and tricorrelation SNR gains versus signal skewness and kurtosis, respectively, using $\Delta t=(1 / 1024) \mathrm{s}$ and $T=T_{s}=2 \mathrm{~s}$.

higher-order detector SNR gain. The minimum signal skewness necessary for a positive bicorrelation SNR gain is

$$
S=\left(\frac{T_{s}^{2}}{z_{n}^{2}(T \Delta t)}\right)^{1 / 4}
$$

and the minimum signal kurtosis necessary for a positive tricorrelation SNR gain is

$$
K=\left(\frac{T_{s}^{2}}{z_{n}^{2}(T \Delta t)}\right)^{1 / 2}-3
$$
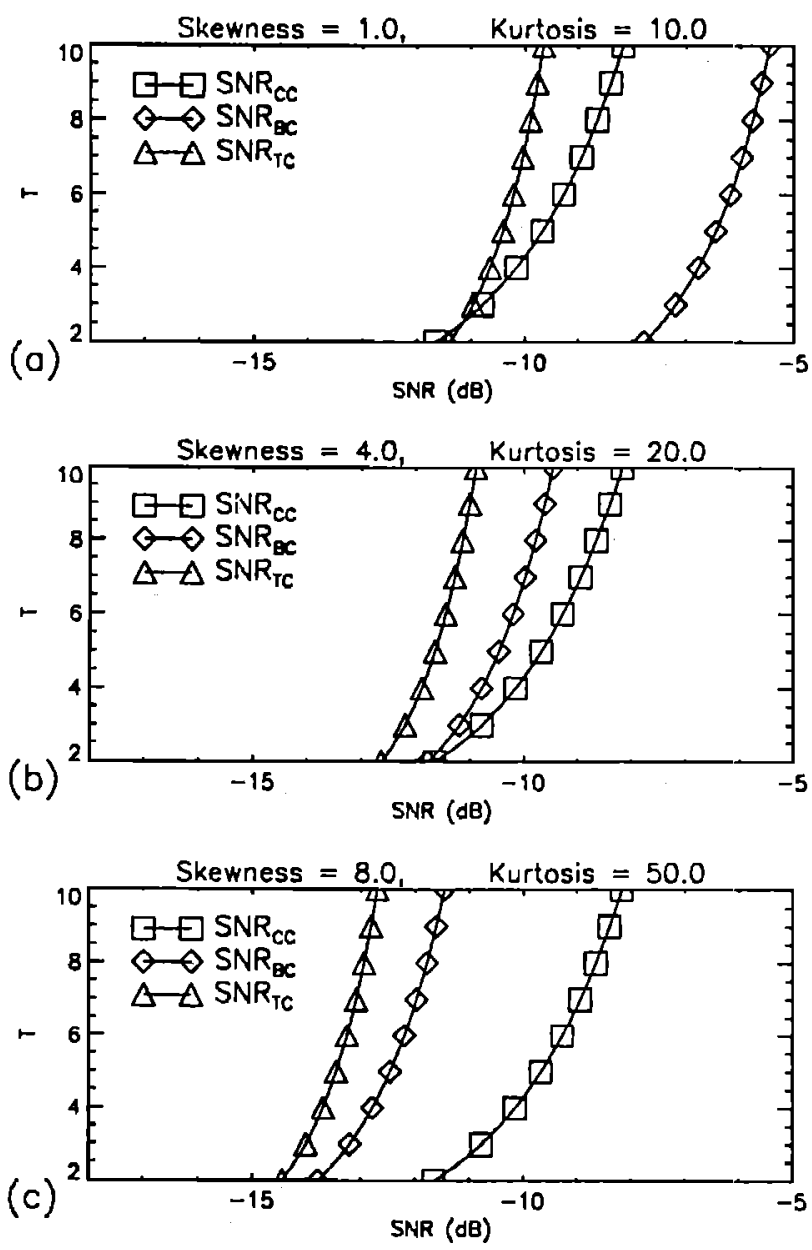

FIG. 2. Theoretical curves of observation time versus SNR required to achieve $P_{d}=0.5$ and $P_{\mathrm{fa}}=0.00 \mathrm{l}$ for the cross-correlation, bicorrelation, and tricorrelation detectors with three 2-s signals of varying skewness and kurtosis. The sampling interval is fixed at $\Delta t=(1 / 1024) \mathrm{s}$.
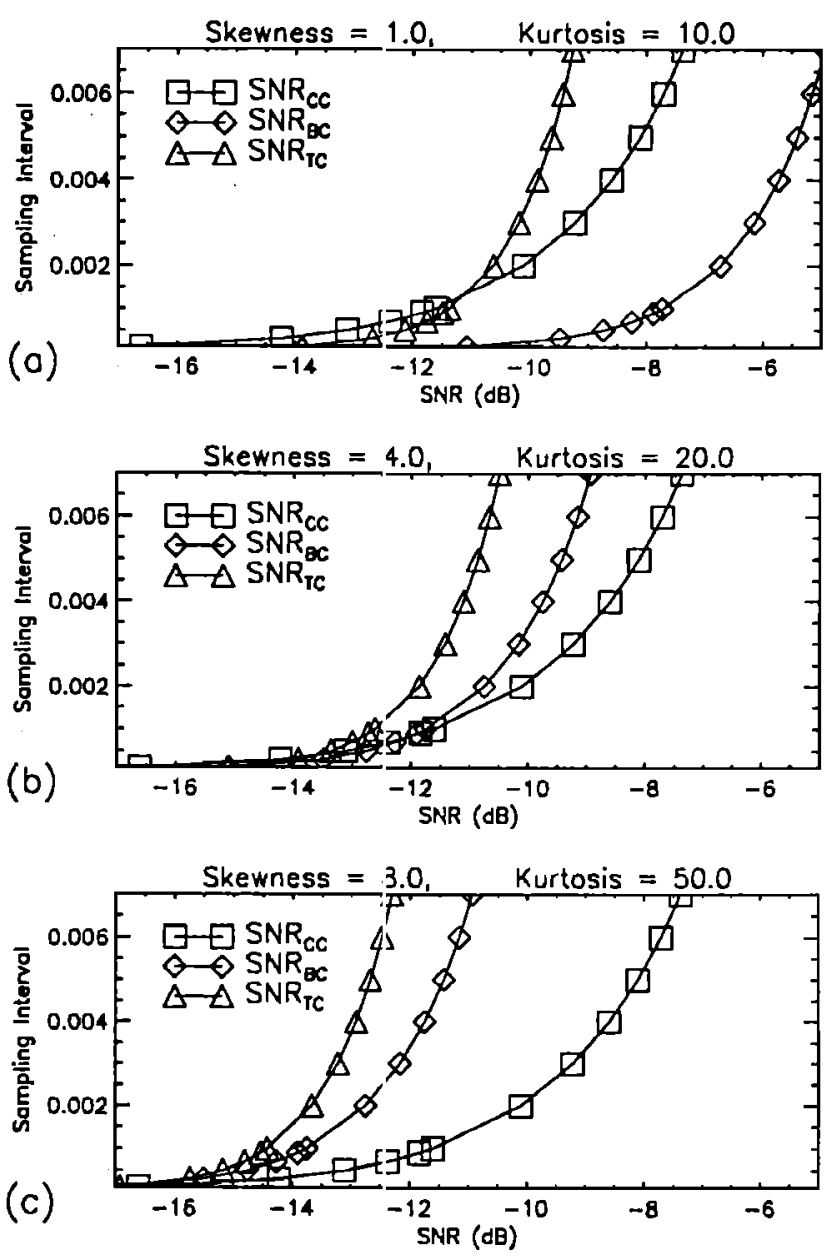

FIG. 3. Theoretical curves of sampling interval versus SNR required to achieve $P_{d}=0.5$ and $P_{\mathrm{ra}}=0.001$ fo: the cross-correlation, bicomelation, and tricorrelation detectors with three '.-s signals of varying skewness and kurtosis. The observation time is fixe $\mid$ at $T=2 \mathrm{~s}$.

For the conditions set in Fig. 1, the minimum signal skewness for a positive bicorrelation SNR gain is 3.83 for $P_{\mathrm{fa}}=0.001$, and 4.41 for $P_{\mathrm{fa}}=0.01$. For the tricorrelation, the minimum signal kurtosis for a positive SNR gain is 11.65 for $P_{\mathrm{fa}}=0.001$, and 16.42 for $P_{\mathrm{fa}}=0.01$.

All of the predictions g.ven in this paper could be given in terms of moments, or moments normalized by the time duration, without considering normalization by powers of the variance, the latter being the commonly used approach for power signals. Because readers may be very familiar with

TABLE I. Normalized moments of the nine test signals. Mean is in amplitude units and variance is in ampli.ude units squared.

\begin{tabular}{ccccc}
\hline \hline Signal number & Mean & Variance & Skewness & Kurtosis \\
\hline 1 & $3.2748 \times 10^{-2}$ & $2.6850 \times 10^{-3}$ & 1.3762 & 0.35895 \\
2 & $2.6776 \times 10^{-2}$ & $2.3151 \times 10^{-3}$ & 1.7013 & 1.4017 \\
3 & $1.8987 \times 10^{-2}$ & $1.7788 \times 10^{-3}$ & 2.2651 & 3.7804 \\
4 & $1.4551 \times 10^{-2}$ & $1.4264 \times 10^{-3}$ & 2.7400 & 6.2895 \\
5 & $9.8513 \times 10^{-3}$ & $1.0117 \times 10^{-3}$ & 3.5188 & 11.459 \\
6 & $4.9714 \times 10^{-3}$ & $5.3476 \times 10^{-4}$ & 5.2247 & 27.271 \\
7 & $1.9937 \times 10^{-3}$ & $2.2039 \times 10^{-4}$ & 8.5062 & 75.059 \\
8 & $9.9722 \times 10^{-4}$ & $1.1124 \times 10^{-4}$ & 12.146 & 154.81 \\
9 & $4.3883 \times 10^{-4}$ & $4.9193 \times 10^{-5}$ & 18.412 & 357.89 \\
\hline \hline
\end{tabular}


TABLE II. Theoretical predictions and computer simulations of SNR $\mathrm{RC}_{\mathrm{C}}$ at $P_{\mathrm{fa}}=0.001$ for the nonzero-mean test signals.

\begin{tabular}{cccc}
\hline \hline & $\begin{array}{c}\text { Theoretical } \\
\text { prediction } \\
\text { SNR }(\mathrm{dB})\end{array}$ & $\begin{array}{c}\text { Computer } \\
\text { calculation } \\
\text { SNR }(\mathrm{dB})\end{array}$ & $\begin{array}{c}\text { Difference } \\
(\mathrm{dB})\end{array}$ \\
\hline 1 & -13.117 & -12.965 & 0.151 \\
2 & -12.829 & -12.688 & 0.141 \\
3 & -12.459 & -12.294 & 0.165 \\
4 & -12.258 & -11.975 & 0.118 \\
5 & -12.055 & -11.911 & 0.144 \\
6 & -11.853 & -11.399 & 0.454 \\
7 & -11.735 & -11.501 & 0.265 \\
8 & -11.696 & -11.500 & 0.196 \\
9 & -11.674 & -11.544 & 0.130 \\
\hline \hline
\end{tabular}

TABLE III. Theoretical predictions and computer simulations of $\mathrm{SNR}_{\mathbf{B C}}$ at $P_{\mathrm{fa}}=0.001$ for the nonzero-mean test signals.

\begin{tabular}{cccc}
\hline \hline & $\begin{array}{c}\text { Theoretical } \\
\text { prediction } \\
\text { SNR (dB) }\end{array}$ & $\begin{array}{c}\text { Computer } \\
\text { calculation } \\
\text { SNR (dB) }\end{array}$ & $\begin{array}{c}\text { Difference } \\
\text { (dB) }\end{array}$ \\
\hline 1 & -11.419 & -11.229 & 0.190 \\
2 & -11.434 & -11.166 & 0.268 \\
3 & -11.565 & -11.314 & 0.251 \\
4 & -11.750 & -11.517 & 0.233 \\
5 & -12.112 & -11.864 & 0.248 \\
6 & -12.900 & -12.643 & 0.257 \\
7 & -14.104 & -14.093 & 0.012 \\
8 & -15.068 & -14.864 & 0.204 \\
9 & -16.235 & -16.048 & 0.187 \\
\hline \hline
\end{tabular}

TABLE IV. Theoretical predictions and computer simulations of $S N_{\mathrm{TC}}$ at $P_{\mathrm{fa}}=0.001$ for the nonzero-mean test signals.

\begin{tabular}{cccc}
\hline \hline & $\begin{array}{c}\text { Theoretical } \\
\text { prediction } \\
\text { SNR }(\mathrm{dB})\end{array}$ & $\begin{array}{c}\text { Computer } \\
\text { calculation } \\
\text { SNR }(\mathrm{dB})\end{array}$ & $\begin{array}{c}\text { Difference } \\
(\mathrm{dB})\end{array}$ \\
\hline 1 & -10.693 & -10.395 & 0.298 \\
2 & -10.862 & -10.637 & 0.225 \\
3 & -11.246 & -11.043 & 0.203 \\
4 & -11.624 & -11.291 & 0.333 \\
5 & -12.268 & -12.129 & 0.139 \\
6 & -13.552 & -13.399 & 0.152 \\
7 & -15.417 & -15.067 & 0.350 \\
8 & -16.882 & -16.654 & 0.228 \\
9 & -18.643 & -18.218 & 0.425 \\
\hline \hline
\end{tabular}

TABLE V. Non-normalized signal moments of the original test signals. Moments are in powers of amplitude units times time in seconds.

\begin{tabular}{cccc}
\hline \hline Signal number & $m_{2}^{s}$ & $m_{3}^{s}$ & $m_{4}^{s}$ \\
\hline 1 & $7.5149 \times 10^{-3}$ & $9.8077 \times 10^{-4}$ & $1.3545 \times 10^{-4}$ \\
2 & $6.0640 \times 10^{-3}$ & $7.8932 \times 10^{-4}$ & $1.0888 \times 10^{-4}$ \\
3 & $4.2787 \times 10^{-3}$ & $5.5622 \times 10^{-4}$ & $7.6679 \times 10^{-5}$ \\
4 & $3.2763 \times 10^{-3}$ & $4.2581 \times 10^{-4}$ & $5.8693 \times 10^{-5}$ \\
5 & $2.2175 \times 10^{-3}$ & $2.8817 \times 10^{-4}$ & $3.9719 \times 10^{-5}$ \\
6 & $1.1190 \times 10^{-3}$ & $1.4541 \times 10^{-4}$ & $2.0042 \times 10^{-5}$ \\
7 & $4.4875 \times 10^{-4}$ & $5.8314 \times 10^{-5}$ & $8.0376 \times 10^{-6}$ \\
8 & $2.2446 \times 10^{-4}$ & $2.9168 \times 10^{-5}$ & $4.0203 \times 10^{-6}$ \\
9 & $9.8771 \times 10^{-5}$ & $1.2835 \times 10^{-5}$ & $1.7691 \times 10^{-6}$ \\
\hline
\end{tabular}

TABLE VI. Non-normalized moments of the zero-mean test signals. Moments are in powers of amplitude units times time in seconds.

\begin{tabular}{cccc}
\hline \hline Signal number & $m_{2}^{s}$ & $m_{3}^{s}$ & $m_{4}^{s}$ \\
\hline 1 & $5.3701 \times 10^{-3}$ & $3.8295 \times 10^{-4}$ & $4.8432 \times 10^{-5}$ \\
2 & $4.6300 \times 10^{-3}$ & $3.7900 \times 10^{-4}$ & $4.7340 \times 10^{-5}$ \\
3 & $3.5577 \times 10^{-3}$ & $3.3988 \times 10^{-4}$ & $4.2910 \times 10^{-5}$ \\
4 & $2.8529 \times 10^{-3}$ & $2.9511 \times 10^{-4}$ & $3.7803 \times 10^{-5}$ \\
5 & $2.0234 \times 10^{-3}$ & $2.2646 \times 10^{-4}$ & $2.5990 \times 10^{-5}$ \\
6 & $1.0695 \times 10^{-3}$ & $1.2921 \times 10^{-4}$ & $1.7313 \times 10^{-5}$ \\
7 & $4.4080 \times 10^{-4}$ & $5.5662 \times 10^{-5}$ & $7.5831 \times 10^{-6}$ \\
8 & $2.2247 \times 10^{-4}$ & $2.8500 \times 10^{-5}$ & $3.9053 \times 10^{-6}$ \\
9 & $9.8385 \times 10^{-5}$ & $1.2705 \times 10^{-5}$ & $1.7467 \times 10^{-6}$ \\
\hline \hline
\end{tabular}

moments normalized by the variance, like skewness and kurtosis, we give prediction formulas for zero-mean signals in terms of skewness and kurtosis.

\section{SAMPLING INTERVAL AND OBSERVATION TIME}

Detection dependence on the number of sample points for deterministic energy signals or stationary power signals has been previously discussed by Giannakis and Tsatsanis (1990), Hinich and Wilson (1990), and Pflug et al. (1992b). Hinich (1990) has shown that the bispectral detector performs well for signals of large time-bandwidth product. In
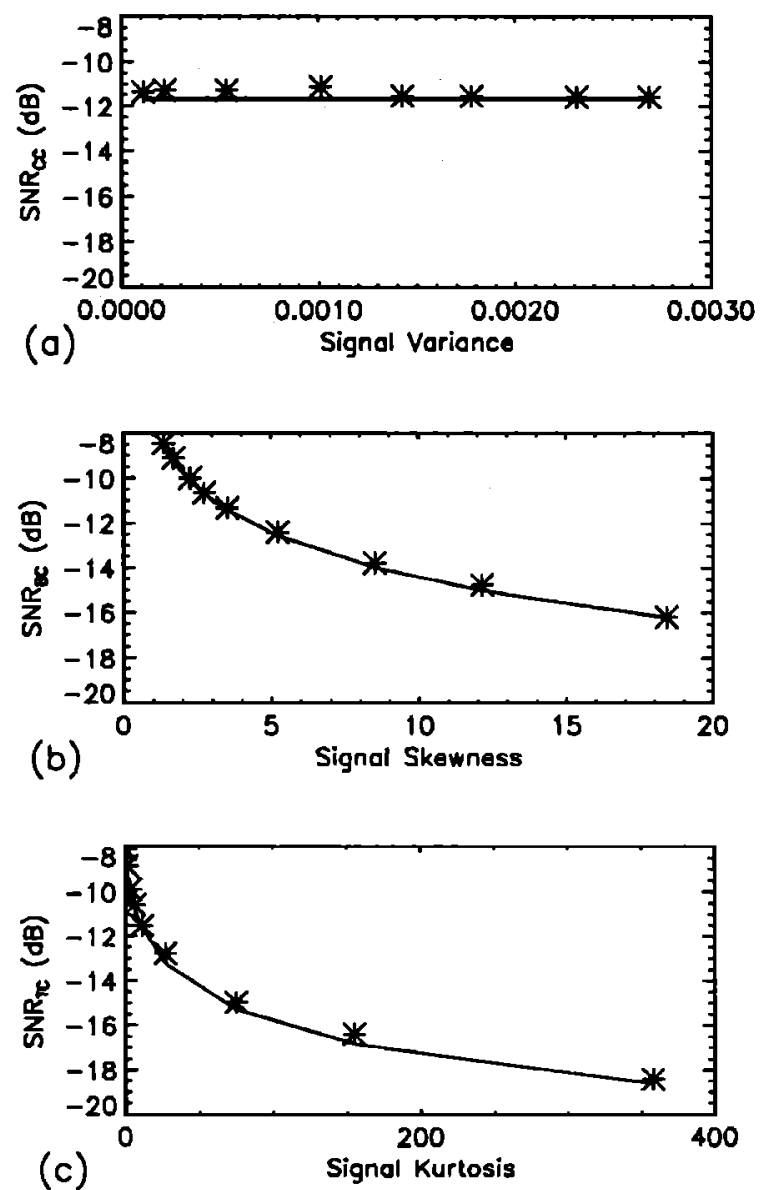

FIG. 4. Theoretical and computer simulation results for (a) $\mathrm{SNR}_{\mathrm{CC}}$, (b) $\mathrm{SNR}_{\mathrm{BC}}$, and (c) $\mathrm{SNR}_{\mathrm{TC}}$ versus normalized signal moments at $P_{\mathrm{fa}}=0.001$ for the zero-mean test signals with $\Delta t=(1 / 1024) \mathrm{s}$ and for $T=2 \mathrm{~s}$. 
the paper by Pflug et al. (1992b), it was shown that for broadband pulse-like signals of relatively large timebandwidth product, the bicorrelation and tricorrelation detectors perform well. However, for highly oscillatory signals they do not. Consideration of only the time-bandwidth product and not the moments is not sufficient for prediction of energy signal detection performance. For energy signals, it is also important to distinguish between the two potential sources of change in the number of sample points, the observation time and the sampling interval.

The SNR for which a correlation detector produces $P_{d}=0.5$ at a fixed $P_{\mathrm{fa}}$ depends on both the sampling interval and the observation time. An increase in $T$ or $\Delta t$ results in an increase in $S N R_{C C}, S N R_{B C}$, and $S N R_{T C}$ as given by Eqs. (28), (31), and (33). The dependence is shown in Figs. 2 and 3. Figure 2 depicts the predicted SNR required to achieve $P_{d}=0.5$ and $P_{\mathrm{fa}}=0.001$ for the cross-correlation, bicorrelation, and tricorrelation detectors for 2-s duration zero-mean signals with skewness and kurtosis pairs of 1.0 and 10.0, 4.0 and 20.0 , and 8.0 and 50.0, respectively. The sampling interval is held constant at $(1 / 1024) \mathrm{s}$, and the variable $T$ is plotted versus $\mathrm{SNR}_{\mathrm{CC}}, \mathrm{SNR}_{\mathrm{BC}}$, and $\mathrm{SNR}_{\mathrm{TC}}$. For all three detectors, as $T$ increases, the SNR for which the detector performs with $P_{d}=0.5$ and $P_{\mathrm{fa}}=0.001$ increases, implying degraded detection capability. However, the relative performance of the higher-order detectors to the cross-correlation detector improves with increasing values of $T$. In Fig. 3, the observation time is fixed at $T=2 \mathrm{~s}$ and the sampling interval is varied. Increasing the sampling interval corresponds to increasing $\mathrm{SNR}_{\mathrm{CC}}, \mathrm{SNR}_{\mathrm{BC}}$, and $\mathrm{SNR}_{\mathrm{TC}}$, and thus degraded detection performance. The different curvatures in the $S N R_{C C}, S N R_{B C}$, and $S N R_{T C}$ curves shown in Figs. 2 and 3 reflect the proportionality of $S N R_{C C}, S N R_{B C}$, and $S N R_{T C}$ to the fourth root, sixth root, and eighth root, respectively, of observation time and sampling interval. As $T$ or $\Delta t$ is increased, the higher-order detectors improve relative to the ordinary correlation detector.

\section{COMPUTER-SIMULATED SNR GAIN CURVES}

In this section, computer simulations are used for comparison with the theoretical gain prediction curves. To create a set of test signals, a 2 -s cosine is amplitude modulated with a set of Gaussian envelopes of decreasing standard deviation resulting in a set of pulse-like transients of the form

$$
x(t)=\cos \left[\pi\left(t-T_{s} / 2\right)\right] e^{-\left[\pi\left(t-T_{s} / 2\right)\right]^{2} / 2 \sigma^{2}} .
$$

As the standard deviation of the Gaussian envelope decreases, the width of the resulting pulse decreases relative to the time window, and the skewness and kurtosis both increase. Nine different test signals were used in the simulations, each with duration $T_{s}=2 \mathrm{~s}$ and sampling interval $=(1 /$ 1024) s. The normalized moments of the test signals are given in Table I. The standard deviation for the Gaussian envelope used to create the pulselike signal must be limited to a minimum value since the sampling interval of $(1 / 1024) \mathrm{s}$ determines signal cutoff frequencies beyond which aliasing occurs in correlations calculated from sampled data. For this sampling interval, $512,341.3$, and $256 \mathrm{~Hz}$ are the frequency cutoffs beyond which the cross-correlation, bicorrelation, and tricorrelation, respectively, would be aliased (Pflug et al., 1992a, 1993; Nielson, 1992; Le Roux et al., 1993). These numbers double if th: same signals are sampled with 2048 points per second. Ths: signals are designated with integers 1-9 such that the high er integers correspond to signals created using narrower Gaissian envelopes. All test signal autobicorrelation peaks occur at zero time lag; hence the bicorrelation threshold detecter will perform better at the central ordinate than at any othe $r$ time lag for each signal (Pflug et al., 1992b).

Although SNR will derrend on the $T_{s}$ chosen, $\mathrm{SNR}_{\mathrm{CC}}$, $S N R_{B C}$, and $S N R_{\text {TC }}$ have the same functional dependence on $T_{s}$, and relative detection performance does not change as a function of $T_{s}$. To illustrate specifically the dependence of detection on signal moment: alone and to facilitate study of the roles that the observation time and sampling interval play in detection, we hold $T_{s}$ cons.tant and large enough to include all values of the broadest test signal considered. This choice means that for the narrowes: signals in our study, there will be a sizable number of leading and trailing negligibly small signal values. If we had been studying these narrower signals individually, smaller values of $T_{s}$ could have been selected.

The test transients are used to create curves of $P_{d}$ versus SNR in a zero-mean Gaussian noise environment at fixed $P_{\mathrm{fa}}=0.001$ and $P_{\mathrm{fa}}=0.01$ fisr each of the three detectors. Gaussian noise is among the noise types that lead to Gaussian distributed correlation central ordinate values which correspond to the assumptions made in Appendixes A and B for derivation of the prediction formulas. Interpolation is used to extract the SNR value for each detector corresponding to $P_{d}=0.5$, the minimum detec able level. Since the signals are nonzero mean, the forms for $S N R_{C C}, S N R_{B C}$, and $S N R_{T C}$ given in Eqs. (28), (31), and (33) are used to predict the SNR required to achieve the minimum detectable level. The theoretical predictions and the results of the computer simulations are given in Tables II, III, and IV. The computer calculations match the theoretical predictions quite well, with a maximum difference in SNR of $0.454 \mathrm{~dB}$ for the crosscorrelation detector, $0.187 \mathrm{~dB}$ for the bicorrelation detector, and $0.425 \mathrm{~dB}$ for the tricorrelation detector.

The non-normalized signal moments, which define the means of the PDFs of the signal-present correlation ensemble and ultimately detec ion performance, change when the mean is subtracted. This is illustrated by comparison of the original non-normalized nonzero-mean signal moments, given in Table $V$, to the non-normalized moments of the test signals with the mean subtracted, given in Table VI. As expected, the signals with larger mean (see Table I) show larger changes in non-normalized signal moments when the mean is removed, and detection performance can change significantly with a small change in signal mean. As an example, for Signal 1, which has a mean value of $3.2748 \times 10^{-2}$ and a maximum amplitude of $0.15 \%$, the nonzero-mean theoretical $\mathrm{SNR}_{\mathrm{CC}}, \mathrm{SNR}_{\mathrm{BC}}$, and $\mathrm{SNR}_{\mathrm{TC}}$ are $-13.117,-11.419$, and $-10.693 \mathrm{~dB}$, respectively, as given in Tables II, III, and IV. Contrast these values with the theoretical $S_{N R}, S_{C C} R_{B C}$, and $\mathrm{SNR}_{\mathrm{TC}}$ values of $-11.157,-8.696$, and $-8.460 \mathrm{~dB}$, 

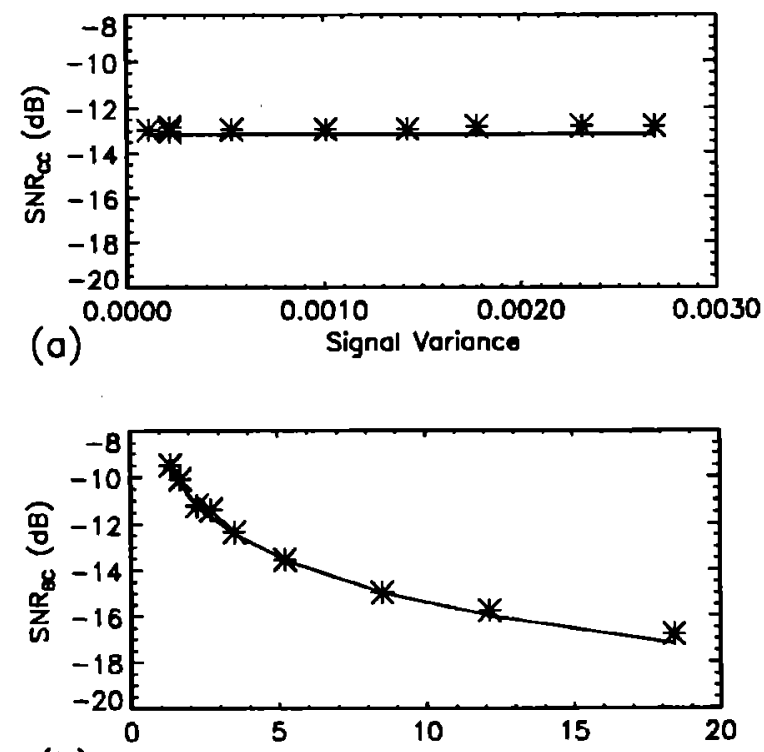

(b)

Signal Skewness

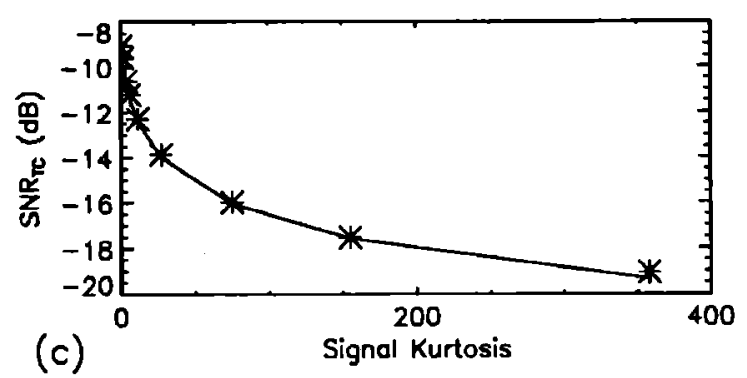

FIG. 5. Theoretical and computer-simulation results for (a) $\mathrm{SNR}_{\mathrm{CC}}$, (b) $\mathrm{SNR}_{\mathrm{BC}}$, and (c) $\mathrm{SNR}_{\mathrm{TC}}$ versus normalized signal moments at $P_{\mathrm{fa}}=0.001$ for the zero-mean test signals with $\Delta t=(1 / 2048) \mathrm{s}$ and for $T=2 \mathrm{~s}$.

respectively, using moments of the zero-mean signal 1 in Eqs. (34)-(36).

To test further the zero-mean signal formulas, simulations were performed for the zero-mean test signals with $T=T_{s}=2 \mathrm{~s}$, and two different sampling intervals, (1/1024) and $(1 / 2048) \mathrm{s}$. For $P_{\mathrm{fa}}=0.001$, the results are shown plotted against the theoretical results in Figs. 4 and 5. The corresponding results for $P_{\mathrm{fa}}=0.01$ are shown in Figs. 6 and 7. In all cases, the simulations match the theoretical predictions closely. As predicted, decreasing the sampling interval results in decreasing levels of $\mathrm{SNR}_{\mathrm{CC}}, \mathrm{SNR}_{\mathrm{BC}}$, and $\mathrm{SNR}_{\mathrm{TC}}$, corresponding to improved detection performance. As shown in the figures, for zero-mean signals, $\mathrm{SNR}_{\mathrm{CC}}$ is independent of the variance of the signal.

Theoretical bicorrelation and tricorrelation SNR gains calculated using Eqs. (37) and (39) and the results of the computer simulations are shown in Figs. 8 and 9 for the test signals with sampling intervals of $(1 / 1024)$ and $(1 / 2048) \mathrm{s}$ for $P_{\mathrm{fa}}=0.001$ and in Figs. 10 and 11 for $P_{\mathrm{fa}}=0.01$. As indicated in the prediction formulas for SNR gain, decreasing the sampling interval decreases the bicorrelation and tricorrelation SNR gains over the cross-correlation detector, even though decreasing the sampling interval improves detection performance overall for each of the three detectors. Decreasing the $P_{\mathrm{fa}}$ (increasing $z_{n}$ ) results in larger bicorrelation and tricorrelation SNR gains; thus higher-order correlators per-
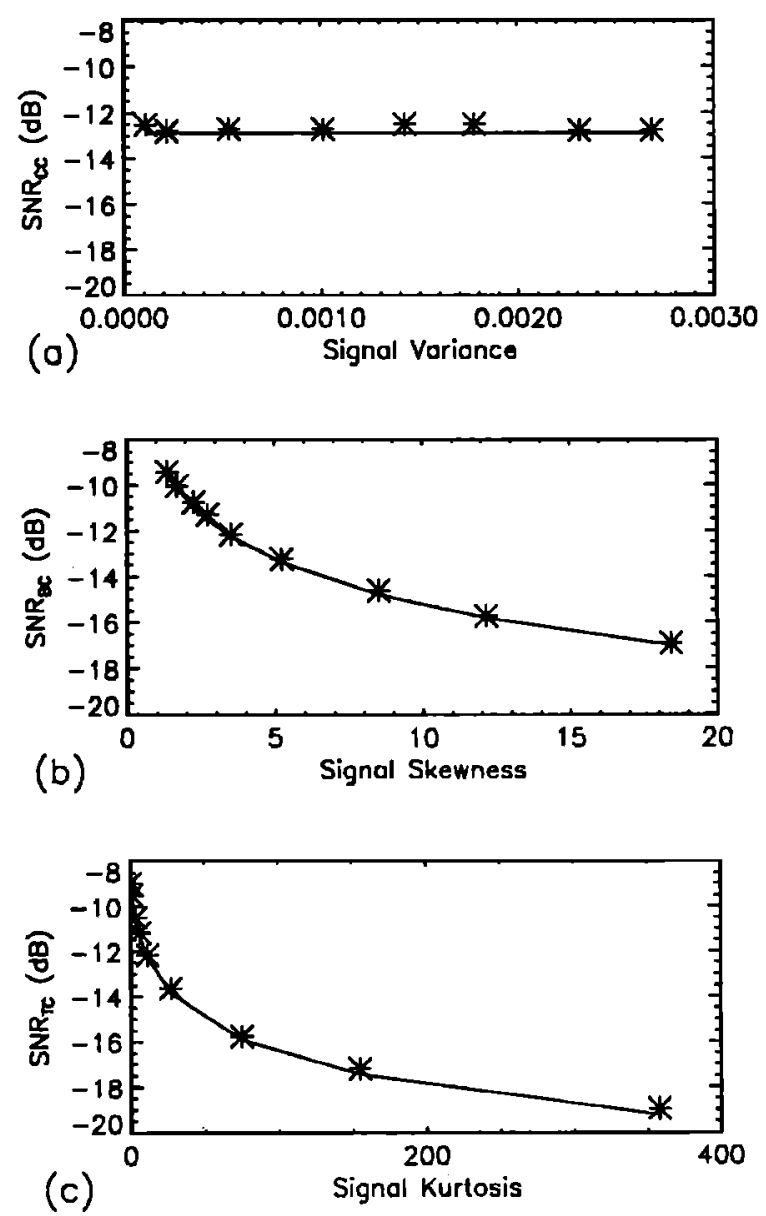

FIG. 6. Theoretical and computer-simulation results for (a) $\mathrm{SNR}_{\mathrm{CC}}$, (b) $\mathrm{SNR}_{\mathrm{BC}}$, and (c) $\mathrm{SNR}_{\mathrm{TC}}$ versus normalized signal moments at $P_{\mathrm{fa}}=0.01$ for the zero-mean test signals with $\Delta t=(1 / 1024) \mathrm{s}$ and $T=2 \mathrm{~s}$.

form at their relative best when the tolerance for false alarm is low.

To test whether the theoretical formulas predict accurately for various observation times, detection performance is evaluated using computer simulations for the 2-s Signal 1 in a 4-s observation window $\left(T_{s}=2 \mathrm{~s}\right.$ and $\left.T=4 \mathrm{~s}\right)$. The results of the computer simulations are $\mathrm{SNR}_{\mathrm{CC}}=-11.311$, $\mathrm{SNR}_{\mathrm{BC}}=-10.134$, and $\mathrm{SNR}_{\mathrm{TC}}=-9.8256 \mathrm{~dB}$. These numbers are close to the theoretical predictions of -11.611 , -10.415 , and $-9.9403 \mathrm{~dB}$, respectively. Similarly, using $T=6 \mathrm{~s}$ for the same signal, the computer simulations result in $\mathrm{SNR}_{\mathrm{CC}}=-10.326, \mathrm{SNR}_{\mathrm{BC}}=-9.4610$, and $\mathrm{SNR}_{\mathrm{TC}}=-9.2428$ $\mathrm{dB}$, compared to the theoretical predictions of -10.731 , -9.8284 , and $-9.4912 \mathrm{~dB}$, respectively.

To predict or simulate performance accurately for very small values of $P_{\mathrm{fa}}$ is generally difficult. The main problem is knowing realistically the shape of the probability density function for the noise and therefore its correlation central ordinate values of interest in the very small tail region. When measured noise statistics are known sufficiently, the domain of the noise probability density function can be well enough determined to do low $P_{\mathrm{fa}}$ performance modeling by theoretical prediction (if formulas are known) or computer simulation. If the actual noise statistics are not sufficiently well known, then inaccuracy is due to experimental limitations and not theoretical definitions. 

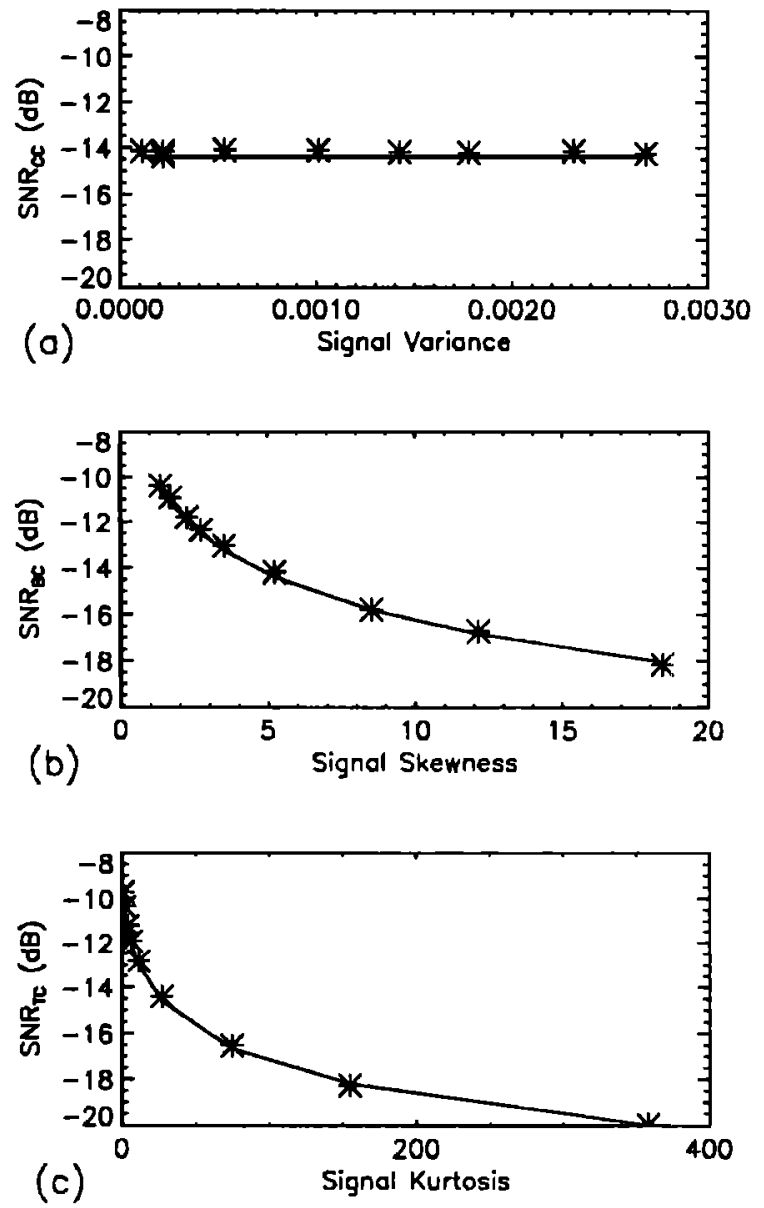

FIG. 7. Theoretical and computer-simulation results for (a) $\mathrm{SNR}_{\mathrm{CC}}$, (b) $\mathrm{SNR}_{\mathrm{BC}}$, and (c) $\mathrm{SNR}_{\mathrm{TC}}$ versus normalized signal moments at $P_{\mathrm{fa}}=0.01$ for the zero-mean test signals with $\Delta t=(1 / 2048) \mathrm{s}$ and for $T=2 \mathrm{~s}$.
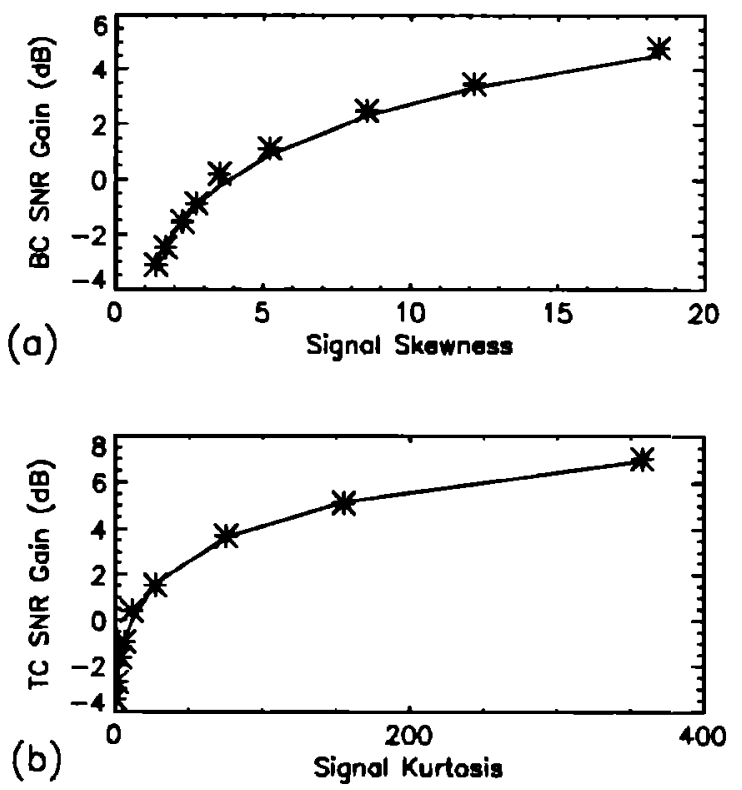

FIG. 8. Theoretical and computer simulation results for (a) bicorrelation SNR gain versus signal skewness and (b) tricorrelation SNR gain versus signal kurtosis at $P_{\mathrm{fa}}=0.001$ for the zero-mean test signals with $\Delta t=(1 /$ 1024) $\mathrm{s}$ and $T=2 \mathrm{~s}$.
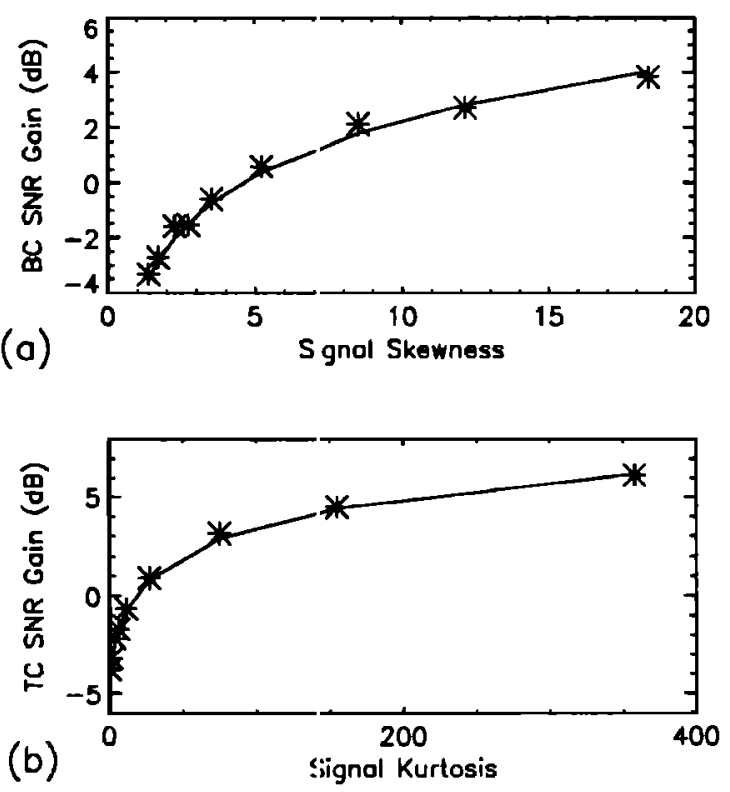

FIG. 9. Theoretical and computt r-simulation results for (a) bicorrelation SNR gain versus signal skewnes: and (b) tricorrelation SNR gain versus signal kurtosis at $P_{\mathrm{fa}}=0.001$ for the zero-mean test signals with $\Delta t=(\mathrm{l} /$ 2048) $\mathrm{s}$ and $T=2 \mathrm{~s}$.

Determining performance for very low $P_{\text {fa }}$ by simulation, however, has two addlitional problems which must be taken into account. The first is that the occurrence of central ordinate values which give a false alarm is so infrequent as to require a huge number of realizations in the simulation to accomplish statistical significance. This can become a prohibitive use of computer tinee. It is also true that many methods used to generate rando $n$ statistical density functions do not produce outliers beyond a certain limiting value and so cannot accurately reproduce the extreme tail values of the density function. For example, the Gaussian noise generator used in our simulations produces a maximum outlier of six for a standard deviation of one.

\section{CONCLUSIONS}

Theoretical formulas are derived for determining crosscorrelation, bicorrelation, and tricorrelation passive detector performance for bandlimited energy transients in zero-mean noise with Gaussian distributed signal-absent correlation central ordinate values. Sptcifically, formulas which predict the SNR required by each of the three detectors to achieve a predetermined level of detection, $P_{d}=0.5$, for any value of $P_{\mathrm{f}_{1}}$, are given. From thesi2, SNR gain formulas based on fixed $P_{d}=0.5$ and variabls: $P_{\mathrm{fa}}$ are derived. The formulas simplify for zero-mean energy signals, and can be expressed using the familiar concepts of signal skewness and kurtosis. For a given signal, knowledge of the low-order signal moments and noise variance are necessary to predict detector performance. For energy signals detector performance depends on $P_{\text {ia }}$, sampling interval, and observation time. Higher-order gain improves with decreasing $P_{\text {fa }}$ and with increasing observation time and sampling interval. Computer 

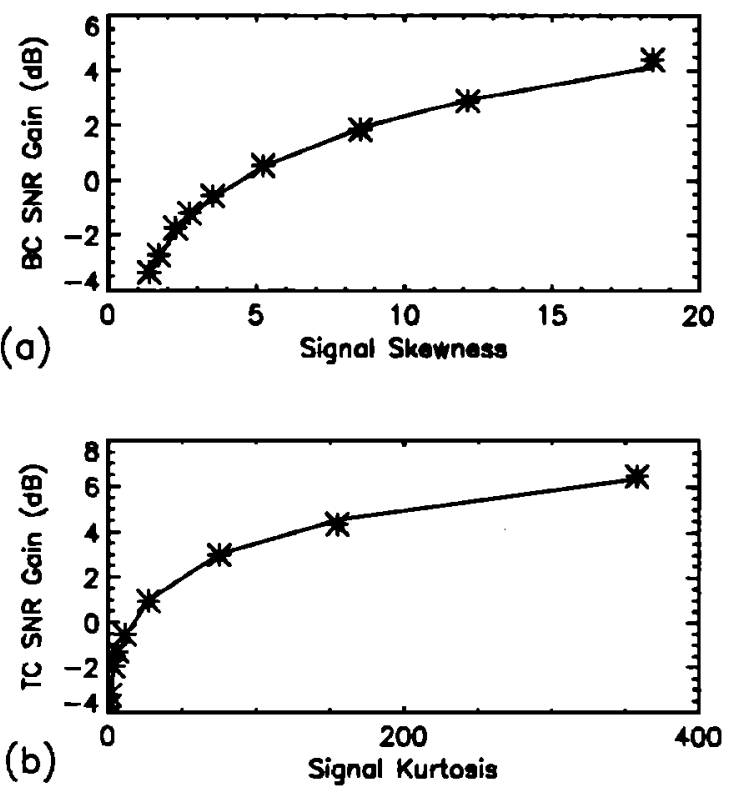

FIG. 10. Theoretical and computer simulation results for (a) bicorrelation SNR gain versus signal skewness and (b) tricorrelation SNR gain versus signal kurtosis at $P_{\mathrm{fa}}=0.01$ for the zero-mean test signals with $\Delta t=(1 / 1024)$ $\mathrm{s}$ and $T=2 \mathrm{~s}$.

calculations using Monte Carlo simulations and hypothesis testing are presented to corroborate the SNR prediction formulas.

\section{ACKNOWLEDGMENTS}

The authors wish to acknowledge support and funding from ONR/NRL Acoustics Transient Core Project No. 715059-0-3, Program Manager Dr. Edward R. Franchi, and ONR/NOARL Grant No. N00014-89-J-6002, and thank M. K. Broadhead, NRL, for insightful discussions and comments on how to improve the manuscript. NRL contribution number NRL/JA/7176--93-0022.
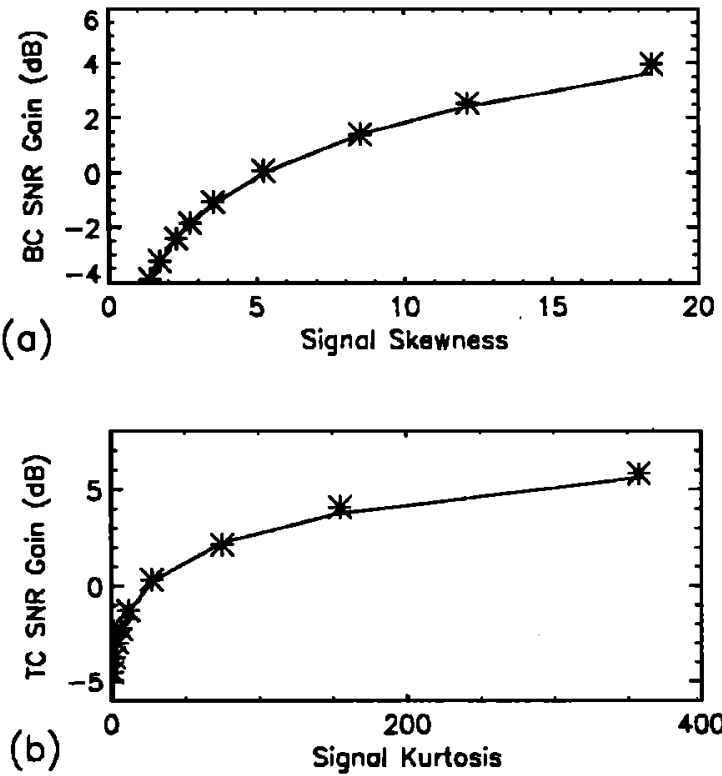

FIG. 11. Theoretical and computer simulation results for (a) bicorrelation SNR gain versus signal skewness and (b) tricorrelation SNR gain versus signal kurtosis at $P_{\mathrm{fa}}=0.01$ for the zero-mean test signals with $\Delta t=(1 / 2048)$ $\mathrm{s}$ and $T=2 \mathrm{~s}$.

\section{APPENDIX A: NOISE ASSUMPTIONS REQUIRED FOR PREDICTION FORMULA ENSEMBLE VARIANCE DERIVATIONS}

The following assumptions concerning the noise are necessary to derive the expressions for ensemble variance of noise correlation central ordinate values given in Eqs. (23h), $(24 \mathrm{~g}$ ), and (25c) (Papoulis, 1965; Robinson, 1980), with $p$ the correlation order and $M$ the number of time points:

(1) The underlying random noise process is such that ensemble members (realizations) are identically distributed and stationary within the observation time.

(2) The infinite-time sum of the product of $p$ distinct realizations over $t_{1}$ and $t_{2}$ is zero except when $t_{1}=t_{2}$. This is approximately true for large $N$.

$$
\begin{aligned}
& (\Delta t)^{2} \sum_{k_{1}=0}^{N-1} \sum_{k_{2}=0}^{N-1}\left\{n_{1}\left(t_{1}\right) n_{2}\left(t_{1}\right) \cdots n_{p}\left(t_{1}\right) n_{1}\left(t_{2}\right) n_{2}\left(t_{2}\right) \cdots n_{p}\left(t_{2}\right)\right\} \\
& =(\Delta t)^{2} \sum_{k_{1}=0}^{N-1} \sum_{k_{2}=0}^{N-1}\left[n_{1}\left(t_{1}\right) n_{2}\left(t_{1}\right) \cdots n_{p}\left(t_{1}\right) n_{1}\left(t_{2}\right) n_{2}\left(t_{2}\right) \cdots n_{p}\left(t_{2}\right)\right] \delta\left(t_{2}-t_{1}\right) \\
& =(\Delta t)^{2} \sum_{k_{1}=0}^{N-1} n_{1}^{2}\left(t_{1}\right) n_{2}^{2}\left(t_{1}\right) \cdots n_{p}^{2}\left(t_{1}\right), \quad 1,2, \cdots p
\end{aligned}
$$

(3) The square of the distinct underlying noise process is uncorrelated across $p$ realizations of the ensemble at any given time,

$$
\begin{aligned}
& E\left\{n_{1}^{2}(t) n_{2}^{2}(t) \cdots n_{p}^{2}(t)\right\} \\
& \quad=E\left\{n_{1}(t)\right\} E\left\{n_{2}(t)\right\} \cdots E\left\{n_{p}(t)\right\}, \quad 1,2, \ldots, p \text { distinct. }
\end{aligned}
$$

Assumption (3) could be replaced by the stronger assump- tion of statistical independence for the realizations of the noise process (Papoulis, 1965). Independence and ergodicity imply assumption (2) for the infinite sum.

\section{APPENDIX B: GAUSSIAN CHARACTER OF CORRELATION CENTRAL ORDINATE PDFS}

Following Isserlis (1918) and Gardner (1986), the $q$ th ensemble moments of zero-mean noise $p$ th-order correlation 
central ordinate PDFs are shown to be consistent with a zeromean Gaussian density. That is, all odd order ensemble moments are zero, and all even order ensemble moments greater than two are appropriately proportional to powers of the second moment.

The assumptions in Appendix A must be extended for the following derivation. A.ssumption 2 must be extended to $q$ time points and assumption 3 must be extended to $q / 2$ time points.

The $q$ th-order ensemt le moment of $p$ zero-mean noise sequences, each of length $N$ with $t_{i}=k_{i} \Delta t$, is

$$
\begin{aligned}
E\{[ & {\left.\left[\sum_{k=0}^{N-1} n_{1}(t) n_{2}(t) \cdots n_{p}(t) \Delta t\right]^{q}\right\} } \\
& =(\Delta t)^{q} E\left\{\left[\sum_{k_{1}=0}^{N-1} n_{1}\left(t_{1}\right) n_{2}\left(t_{1}\right) \cdots n_{p}\left(t_{1}\right)\right]\left[\sum_{k_{2}=0}^{N-1} n_{1}\left(t_{2}\right) n_{2}\left(t_{2}\right) \cdots n_{p}\left(t_{2}\right)\right] \cdots\left[\sum_{k_{q}=0}^{N-1} n_{1}\left(t_{q}\right) n_{2}\left(t_{q}\right) \cdots n_{p}\left(t_{q}\right)\right]\right\} \\
& =(\Delta t)^{q} E\left\{\sum_{k_{1}=0}^{N-1} \sum_{k_{2}=0}^{N-1} \cdots \sum_{k_{q}=1}^{N-1} n_{1}\left(t_{1}\right) n_{1}\left(t_{2}\right) \cdots n_{1}\left(t_{q}\right) n_{2}\left(t_{1}\right) n_{2}\left(t_{2}\right) \cdots n_{2}\left(t_{q}\right) \cdots n_{p}\left(t_{1}\right) n_{p}\left(t_{2}\right) \cdots n_{p}\left(t_{q}\right)\right\} .
\end{aligned}
$$

For large $N$, this expression is approximately zero when $p$ is odd, and whenever all $t_{i}$ are distinct. It is only nonzero when $p$ is even and times are equal in pairs. Using delta function notation, the moments are nonze ro when

$$
\begin{aligned}
(\Delta t)^{q} E\left\{\sum_{k_{1}=0}^{N-1} \sum_{k_{2}=0}^{N-1} \cdots \sum_{k_{q}=0}^{N-1} n_{1}\left(t_{1}\right) n_{1}\left(t_{2}\right) \cdots n_{1}\left(t_{q}\right) n_{2}\left(t_{1}\right) n_{2}\left(t_{2}\right) \cdots n_{2}\left(t_{q}\right) \cdots n_{p}\left(t_{1}\right) n_{p}\left(t_{2}\right) \cdots n_{p}\left(t_{q}\right)\right. \\
\left.\quad \times\left[\sum \delta\left(t_{j_{1}}-t_{j_{2}}\right) \cdots \delta\left(t_{j_{q-1}}-t_{j_{q}}\right)\right]\right\}
\end{aligned}
$$

is nonzero, where the summation over the product of delta functions is taken over all possible ways of dividing $q$ integers into $q / 2$ combinations of pairs. There are $(1)(3)(5) \ldots(q-3)(q-1)$ terms in the summation. Applying the delta summation results in

$$
\begin{aligned}
& {[(1)(3)(5) \cdots(q-3)(q-1)](\Delta t)^{q} N^{q / 2} E\left\{n_{1}^{2}\left(t_{1}\right) n_{1}^{2}\left(t_{2}\right) \cdots n_{1}^{2}\left(t_{q / 2}\right)\right.} \\
& \left.\quad \times n_{2}^{2}\left(t_{1}\right) n_{2}^{2}\left(t_{2}\right) \cdots n_{2}^{2}\left(t_{q / 2}\right) \cdots n_{p}^{2}\left(t_{1}\right) n_{p}^{2}\left(t_{2}\right) \cdots n_{p}^{2}\left(t_{q / 2}\right)\right\} .
\end{aligned}
$$

Since the square of the noise is uncorrelated in time, this is equal to

$$
\begin{aligned}
{[(1)} & (3)(5) \cdots(q-3)(q-1)](\Delta t)^{q} N^{q / 2} E\left\{n_{1}^{2}\left(t_{1}\right)\right\} E\left\{n_{1}^{2}\left(t_{2}\right)\right\} \cdots E\left\{n_{1}^{2}\left(t_{q / 2}\right)\right\} E\left\{n_{2}^{2}\left(t_{1}\right)\right\} \\
& \times E\left\{n_{2}^{2}\left(t_{2}\right)\right\} \cdots E\left\{n_{2}^{2}\left(t_{q / 2}\right)\right\} E\left\{n_{p}^{2}\left(t_{1}\right)\right\} E\left\{n_{p}^{2}\left(t_{2}\right)\right\} \cdots E\left\{n_{p}^{2}\left(t_{q / 2}\right)\right\} \\
= & {[(1)(3)(5) \cdots(q-3)(q-1)](\Delta t)^{q} N^{q / 2}\left[E\left\{n^{2}(t)\right\}\right]^{p q / 2}, }
\end{aligned}
$$

which is the even $q$ th-order ensemble moment of $p$ zeromean noise sequences. These powers of the moments are consistent with the moment relationships for a Gaussian density.

Bracewell, R. N. (1986). The Fourier Transform and Its Applications, 2nd ed., revised (McGraw-Hill, New York).

Egan, J. P. (1975). Signal Detection Theory and ROC Analysis (Academic, New York).

Field, R. L., and Leclere, J. H. (1993). "The effects of a bottom-limited ocean on signal kurtosis-Applications of tricorrelation detection and minimum entropy deconvolution of broadband signals," J. Acoust. Soc. Am. 94, 1868.

Gardner, W. A. (1986). Introduction to Random Processes (Macmillan, New York).

Giannakis, G. B., and Tsatsanis, M. K. (1990). "Signal detection and classification using matched filtering and higher order statistics," IEEE Trans. Acoust. Speech Signal Process. 38, 1284-1296.

Hinich, M. J., and Wilson, G. R. (1990). "Detection of non-Gaussian signals in non-Gaussian noise using the bispectrum," IEEE Trans. Acoust. Speech Signal Process. 38, 1126-1131

Hinich, M. J. (1990). "Detecting a transient signal by bispectral analysis," J. Acoust. Soc. Am. 38, 1277-1233.

Ioup, G. E.. Ioup, J. W., Field, R. L., and Leclere, J. H. (1989a). "Higher order correlations for transient cetection," Proceedings of the First Acoustic Transient Workshop Held at Naval Research Laboratory (formerly NORDA), Stennis Space Center, Naval Research Laboratory Publication No. $152-5100$, pp. $628-660$.

Ioup, G. E., Ioup, J. W., Barnes, K. H., Field, R. L., Leclere, J. H., and Rayborn, G. H. (1989b). "Evalıation of bicorrelations for transient detection," Proceedings of the Workshop on Higher-Order Spectral Analysis, Vail, CO, pp. 46-51.

Ioup, G. E., Pflug, L. A., Ioup, J. W., Barnes, K. H., Field, R. L., Leclere, J. H., and Rayborn, G. H. (1991). "Higher order correlations for the detection of deterministic transients," U.S.N. J. Underwater Acoust. $40925-$ 944.

Ioup, G. E., Pflug, L. A., Ioup, J. W., and Field, R. L. (1993). "Prefiltering for higher order advantage," Proceedings of the IEEE Signal Processing Workshop on Higher-Order St: tistics, South Lake Tahoe, CA, pp. 309313.

Isserlis, L. (1918). " On a formula for the product-moment coefficient of any 
order of a normal frequency distribution in any number of variables," Biometrika 12, 134-139.

Kletter, D., and Messer, H. (1989). "Detection of a non-Gaussian signal in Gaussian noise using higher-order spectral analysis," Proceedings of the Workshop on Higher-Order Spectral Analysis, Vail, CO, pp. 95-99.

Le Roux, J., Christophe, C., and Rossille, D. (1993). "Bispectral analysis and reconstruction in the frequency domain of mono- and bidimensional deterministic sampled signals," Multidimensional Syst. Signal Process. 4, $39-66$.

Nielsen, P. A. (1992). "Comments on: 'Properties of higher-order correlations and spectra for bandlimited deterministic transients,' [J. Acoust. Soc. Am. 91, 975-988 (1992)]," J. Acoust. Soc. Am. 92, 1763-1765.

Papoulis, A. (1965). Probability, Random Variables, and Stochastic Processes (McGraw-Hill, New York).

Pflug, L. A., Barnes, K. H., Ioup, G. E., Ioup, J. W., Field, R. L., Leclere, J. H., and Rayborn, G. H. (1989). "Higher order correlations and spectra and the detection of narrow band deterministic transients," J. Acoust. Soc. Am. Suppl. 186, S118.

Pflug, L. A., Ioup, G. E., Ioup, J. W., Barnes, K. H., Field, R. L., and Leclere, J. H. (1990a). "Effects of environmental degradation on higherorder correlation detectors for deterministic signals," J. Acoust. Soc. Am. Suppl. 1 87, S155.

Pflug, L. A., Ioup, G. E., Ioup, J. W., Barnes, K. H., Field, R. L., and
Rayborn, G. H. (1990b). "Detection and time-delay estimation for bandlimited transients using higher order correlations," J. Acoust. Soc. Am. Suppl. 1 88, $\$ 29$

Pflug, L. A., Ioup, G. E., Ioup, J. W., and Field, R. L. (1992a). "Properties of higher order correlations and spectra for bandlimited, deterministic transients," J. Acoust. Soc. Am. 91, 975-988.

Pflug, L. A., Ioup, G. E., Ioup, J. W., Barnes, K. H., Field, R. L., and Rayborn, G. H. (1992b). "Detection of oscillatory and impulsive transients using higher-order correlations and spectra," J. Acoust. Soc. Am. 91, 2763-2776.

Pflug, L. A., Ioup, G. E., and Ioup, J. W. (1993). "Sampling requirements and aliasing for higher-order correlations," J. Acoust. Soc. Am. 94, 2159 2172.

Pflug, L. A., Ioup, G. E., Ioup, J. W., and Field, R. L. (1994). "Prefiltering for improved correlation detection of bandlimited transient signals," J. Acoust. Soc. Am. 95, 1459-1473.

Press, W. H., Flannery, B. P., Teukolsky, S. A., and Vetterling, W. T. (1986). Numerical Recipes: The Art of Scientific Computing (Press Syndicate of the University of Cambridge, New York).

Robinson, E. A. (1980). Physical Applications of Statistical Time-Series (Macmillan, New York).

Van Trees, H. L. (1968). Detection, Estimation, and Modulation Theory (Wiley, New York). 